\title{
Adjoint-based sensitivity analysis of char combustion surface reaction kinetics
}

\author{
Ahmed Hassan . Taraneh Sayadi · Martin \\ Schiemann - Viktor Scherer
}

Received: date / Accepted: date

\begin{abstract}
Simulations of solid particle combustion rely on models to approximate the reactions on the surface of the particle and in the surrounding gas. These models, in turn, depend on many model parameters, which are determined, most commonly, by experiments and contain a certain level of uncertainty. It is therefore essential to correctly determine the sensitivities of measured quantities of interest, with respect to the existing model parameters. This study, concentrates on a one-step model describing the heterogeneous reaction on the surface of a char particle, and in particular, the surface model presented by Schiemann et al. 24]. Adjoint-based methods are then employed to extract sensitivities of various quantities of interests, including the burning rate and total heat release, with respect to surface model parameters, such as, the activation energy, and the pre-exponential factor. The variation of these sensitivities are then assessed as the particle size and free stream composition are varied.
\end{abstract}

Keywords Char burnout, sensitivity analysis, adjoint-based methods

\section{Introduction}

Simulations of reactive flows are performed by accounting for both transport and chemistry and their interaction. Due to the prohibitive costs of reactive flow computations, arising from the large range of scales, and the equations governing the evolution of all the species, models are employed to help approximate the underlying chemistry. In the case of solid particle combustion, due to the presence of reactions on the surface of the particle, the underlying dynamics are even more complex, and the accuracy of the simulation results are highly dependent on reliable surface reaction models employed.

\author{
Ahmed Hassan \\ Institute for Combustion Technology, RWTH Aachen University, Aachen, Germany \\ Tel.: +1-217-300-0901 \\ E-mail: a.hassan@itv.rwth-aachen.de \\ Taraneh Sayadi \\ Jean le Rond d'Alembert Institute, CNRS/Sorbonne University, Paris, France \\ Institute for Combustion Technology, RWTH Aachen University, Aachen, Germany \\ Martin Schiemann \\ Department of Energy Plant Technology, Ruhr-University Bochum, Germany \\ Viktor Scherer \\ Department of Energy Plant Technology, Ruhr-University Bochum, Germany
}


The most conventional models used to simulate coal particle combustion are the single film [21, 4, double film [27, and continuous film [9] models. These models are mostly developed in onedimensional settings and in the steady state limit. The fidelity of the model can be increased by considering ever more complex gas and particle-phase chemistry [17. Regardless of the fidelity, however, these models generally rely on many parameters in order to approximate the physical problem. These parameters are then determined using experimental conditions resembling typical char combustion environment. Careful selection of experimental conditions, favoring the char reaction process by de-emphasizing heat and mass transfer, has allowed minimising uncertainties in species transport properties [24]. Nevertheless, a certain level of uncertainty still remains in such experimental measurements. Therefore, it is of great importance to determine the sensitivity of the output (final solution or quantity of interest) to the existing parameters in the model.

Most common methods in extracting the gradient information are analytical or use finite differences. The subtleties associated with the numerical approach, such as the meshing process, discretization, and complex governing equations, especially when chemical reactions and regions with steep gradients (flames) are present, make extracting the analytical expression close to impossible. In the case of finite differences, the numerous parameters involved in modeling make this approach prohibitively expensive for large problems and can also be easily overwhelmed by numerical noise. Adjoint-based methodology overcomes these two shortcomings, by allowing the determination of the gradient at a cost comparable to a single function evaluation [7. The backward solution (the solution of the adjoint equation) in effect provides gradient or sensitivity information. Given an objective function (quantity of interest), as well as a set of governing equations and side conditions, using the adjoint solution, a gradient of the quantity of interest with respect to specified input variables (model parameters) can be determined, while the governing equations are satisfied. This gradient is computed in the form of algebraic expressions based on the problem's Lagrange multipliers or adjoint variables, which in turn is used in standard optimization algorithms that rely on Jacobian information (such as the conjugate-gradient family). This approach, although more involved, is gaining traction especially in the field of acoustics and thermoacoustics [13, 15, 6]. Recently, nonlinear problems have also been tackled, within the context of optimal control of separation on a realistic high-lift airfoil or wing, enhancement of mixing efficiency, minimal turbulence seeds and shape optimization $19,25,22,5$. Adjoint-based methods have also been recently used to perform sensitivity analysis with respect to model parameters in reactive flow regimes $[3,23,14,8$.

Previous studies have demonstrated the importance of extracting in situ particle temperature and size data, in order to extract the desired kinetic models, which outperforms the more common approach, relying on bulk measurements. In this study, therefore, we rely on a surface model, extracted using this approach, in order to capture surface reactions of char particle combustion 24], where particle temperature and size data are gathered, and combined with other experimental information, to produce the best fit parameter of the chosen char combustion model. Since heat and mass transfer is described reasonably well with established models, the kinetics parameters of the surface reaction mechanism are those fitted through gathered experimental data. Adjoint-based methods are then employed to extract the sensitivity of the described surface reaction model, with respect to various quantities of interest, to provide a detailed assessment of the existing sensitivities in the model parameters.

The study is structured as follows: The governing equations describing the evolution of species and temperature on the particle surface as well as in the gas phase, together with appropriate boundary conditions are presented in section 2 . The numerical approach is briefly described in section 3. The methodology for developing the adjoint framework is then presented in section 4. The results of the extracted sensitivities are reported in section 5 . Finally, summary and conclusion of this work are discussed in section 6 


\section{Governing equations}

In this section, the governing equations describing flow/chemistry, in the gas phase, and on the particle surface, along with the accompanying boundary conditions, are presented. Throughout the study, the superscript " $g$ " denotes variables defined in the gas phase, and superscript " $s$ " refers to the variables defined on the surface of the particle. Superscript "*" signifies non-dimensional quantities, whenever applicable.

\subsection{Equations describing the gas phase}

Assuming a coal particle, the following homogeneous reaction is considered for the oxidation of carbon monoxide in the gas phase,

$$
\mathrm{CO}+\frac{1}{2} \mathrm{O}_{2} \rightarrow \mathrm{CO}_{2}
$$

The evolution of the gaseous composition is described by the species mass fraction equations,

$$
\nabla \cdot\left(\rho Y_{i}\left(\mathbf{v}+\mathbf{V}_{i}\right)\right)=\omega_{i}
$$

where $i=1, \ldots, 4$ refer to fuel $(\mathrm{CO})$, oxidizer $\left(\mathrm{O}_{2}\right)$, product $\left(\mathrm{CO}_{2}\right)$, and inert gas $\left(\mathrm{H}_{2} \mathrm{O}\right)$, respectively. $\rho$, denotes the mixture density, $\mathbf{v}$, the mass-averaged velocity, and $\mathbf{V}_{i}$ the diffusion velocity for species $i$. The term on the right hand side of the above equation reads $\omega_{i}=\mathcal{W}_{i}\left(\nu_{i}^{\prime \prime}-\nu_{i}^{\prime}\right) \omega$, where $\nu^{\prime \prime}{ }_{i}$ and $\nu_{i}^{\prime}$ are the stoichiometric coefficients of the product and reactants in the homogeneous reaction, and $\mathcal{W}_{i}$ denotes the molecular weight of species $i$. The homogeneous reaction rate obeys Arrhenius law 11, 16,

$$
\begin{aligned}
\omega & =k[\mathrm{CO}]\left[\mathrm{O}_{2}\right]^{\frac{1}{2}}\left[\mathrm{H}_{2} \mathrm{O}\right]^{\frac{1}{2}} \exp \left(-\frac{E_{a}^{g}}{R T}\right) \\
& =\frac{k}{\mathcal{W}_{\mathrm{CO}} \mathcal{W}_{\mathrm{O}_{2}}^{\frac{1}{2}} \mathcal{W}_{\mathrm{H}_{2} \mathrm{O}}^{\frac{1}{2}}} \rho^{2} Y_{1} Y_{2}^{\frac{1}{2}} Y_{4}^{\frac{1}{2}} \exp \left(-\frac{E_{a}^{g}}{R T}\right),
\end{aligned}
$$

where, $T, E_{a}^{g}$, and $R$ denote the gas temperature, the activation energy for the gas reaction, and the universal gas constant, respectively. Using Fick's law, the diffusion velocity for species $i$ can be closed as,

$$
Y_{i} \mathbf{V}_{i}=-D \nabla Y_{i}
$$

where the diffusion coefficient is assumed identical for all species. Substituting Eq. 5 in Eq. 2 leads to the following transport equation for the species,

$$
\nabla \cdot\left(\rho \mathbf{v} Y_{i}-\rho D \nabla Y_{i}\right)=\omega_{i}
$$

Similarly, assuming identical and constant specific heats $C_{p}$ for all species, the energy equation yields the following relation for the temperature in the gas phase,

$$
\nabla \cdot\left(\rho C_{p} \mathbf{v} T-\lambda \nabla T\right)=-\sum_{i=1}^{4} h_{i}^{0} \omega_{i}
$$

where $h_{i}^{0}$ is the enthalpy of formation of species $i$, and $\lambda$ is the coefficient of heat conduction. For low speed and steady flow, viscous effects are often negligible and the momentum equation reduces to the pressure being approximately constant. Finally, the continuity equation holds,

$$
\nabla \cdot(\rho \mathbf{v})=0
$$


For the purpose of this study, $\rho D$ is assumed constant and the Lewis number, Le $=\lambda / \rho D C_{p}$ is set to unity. The variables are made dimensionless by introducing the following characteristic values, as proposed by Matalon [16]: (i) the particle radius a for length, (ii) $Q / C_{p}$ for temperature, where $Q$ is the heat of the homogeneous reaction per unit mass of $\mathrm{CO}_{2}$, namely,

$$
Q=-\frac{\sum_{i=1}^{4} h_{i}^{0} \mathcal{W}_{i}\left(\nu_{i}^{\prime \prime}-\nu_{i}^{\prime}\right)}{\mathcal{W}_{\mathrm{CO}_{2}}\left(\nu_{\mathrm{CO}_{2}}^{\prime \prime}-\nu_{\mathrm{CO}_{2}}^{\prime}\right)},
$$

(iii) $\rho_{0}=p_{0} C_{P} / Q \tilde{R}$, where $\tilde{R}$ is the specific gas constant based on average molecular weight, and $p_{0}$ is the uniform pressure, finally (iv) $\lambda / \rho_{0} C_{p} a$, for velocity. This non-dimensionalisation yields the following expression for the Damköhler number of the homogeneous reaction

$$
\mathrm{Da}^{g}=\frac{k\left(\nu_{\mathrm{CO}_{2}}^{\prime \prime}-\nu_{\mathrm{CO}_{2}}^{\prime}\right) C_{p}^{3}}{\lambda Q^{2} \tilde{R}^{2}} \frac{\mathcal{W}_{\mathrm{CO}_{2}}}{\mathcal{W}_{\mathrm{CO}} \mathcal{W}_{\mathrm{O}_{2}}^{\frac{1}{2}} \mathcal{W}_{\mathrm{H}_{2} \mathrm{O}}^{\frac{1}{2}}} a^{2} p_{0}^{2} .
$$

In non-dimensional form, the equation of state (ideal gas) reads $\rho T=1$. Finally, the non-dimensional equations are:

$$
\begin{aligned}
\forall i \in\{1,4\}, \mathcal{L}\left(Y_{i}\right) & =\Omega \alpha_{i}, \\
\mathcal{L}(T) & =\Omega \alpha_{5},
\end{aligned}
$$

where, $\left(\alpha_{i}\right)_{i \in\{1,5\}}=(\alpha-1,-\alpha, 1,0,1)$ and $\alpha=\mathcal{W}_{\mathrm{O}_{2}} / 2 \mathcal{W}_{\mathrm{CO}_{2}}$,

$$
\mathcal{L}(\varphi)=\nabla \cdot(\rho \mathbf{v} \varphi-\nabla \varphi),
$$

where, $\varphi=\left\{Y_{i}, T^{*}\right\}$, and

$$
\Omega=\mathrm{Da}^{g} \frac{Y_{1} Y_{2}^{\frac{1}{2}} Y_{4}^{\frac{1}{2}}}{T^{* 2}} \exp \left(-\frac{E_{a}^{g *}}{T^{*}}\right) .
$$

If axi-symmetry is assumed, the governing equations reduce to a set of coupled second order ordinary differential equations (ODE). In the case of a single spherical particle in particular, the continuity equation (in spherical coordinates) yields

$$
r^{2} \rho v_{r}=\dot{M}=\text { constant }
$$

where $r$ is the radial coordinate, $v_{r}$ the radial velocity component and $\dot{M}$ denotes the "burning rate". The non-dimensional linear operator $\mathcal{L}$ then reduces to

$$
\mathcal{L}_{\text {spherical }}(\varphi)=\frac{\dot{M}^{*}}{r^{* 2}} \frac{\mathrm{d} \varphi}{\mathrm{d} r^{*}}-\frac{1}{r^{* 2}} \frac{\mathrm{d}}{\mathrm{d} r^{*}}\left(r^{* 2} \frac{\mathrm{d} \varphi}{\mathrm{d} r^{*}}\right),
$$

where, $\dot{M}^{*}=\frac{\lambda}{C_{p} a} \dot{M}$, and $r^{*}=\frac{r}{a}$. In this study, heat capacity is $C_{p}=29.1 \frac{\mathrm{J}}{\text { moleK }}$, and the gas conductivity is $\lambda=39.4 \times 10^{-3} \frac{J}{\text { mole.K.m.s. }}$.

\subsection{Equations at the particle surface}

The particle is assumed isothermal $\left(T=T^{s}\right.$ for $\left.r<1\right)$, and the temperature profile is also assumed continuous, which yields the following boundary condition

$$
T^{*}(r=1)=T^{s} .
$$

The remaining conditions at the particle surface are extracted assuming the following heterogeneous reaction on the surface of the particle [24]:

$$
\mathrm{C}[s]+\left(\frac{1+\psi}{2}\right) \mathrm{O}_{2} \rightarrow(1-\psi) \mathrm{CO}+\psi \mathrm{CO}_{2}
$$


The stoichiometric ratio of oxygen depends on the value of $\psi$, which is determined from the relation given by Tognotti [28. In non-dimensional form the relation is given by,

$$
\frac{\psi}{1-\psi}=0.02 P^{* s}\left(\mathrm{O}_{2}\right)^{0.21} \exp \left(\frac{T_{a} C_{p}}{Q T^{s}}\right)
$$

where, $T_{a}=3070 \mathrm{~K}, P^{*}=\frac{P}{P_{0}}$ is the non-dimensional pressure, and $Q$ is the heat of the homogeneous reaction per unit mass of $\mathrm{CO}_{2}$, defined by equation 9 . The oxygen partial pressure $P^{s}\left(\mathrm{O}_{2}\right)$ is computed from the following expression,

$$
P^{s}\left(\mathrm{O}_{2}\right)=P_{0} \frac{Y_{2}^{s} \bar{W}}{\mathcal{W}_{\mathrm{O}_{2}}}
$$

Due to large solid to gas density ratio, the quasi-steady assumption holds. Conservation statements for each species $i$, which match the differences between the net fluxes of each species to the rates of consumption/production per unit area due to the heterogeneous reaction rates, results in the following mass balance:

$$
\rho \mathbf{v} \cdot \mathbf{n} Y_{i}^{s}-\left.\rho D \nabla Y_{i}\right|^{s} \cdot \mathbf{n}=\omega_{i}^{\prime}
$$

where, $\mathbf{n}$ denotes the outward pointing normal to the particle surface, and $\omega_{i}^{\prime}$ the reaction rate of species i due to the heterogeneous reaction. The non-dimensional and axi-symmetric form of the above equation reads,

$$
\dot{M}^{*} Y_{i}^{s}-\left.\frac{\mathrm{d} Y_{i}}{\mathrm{~d} r^{*}}\right|^{s}=\omega_{i}^{\prime} \frac{C_{p} a}{\lambda} .
$$

The global reaction rate for surface reaction obeys Arrhenius law, and is given as [24,

$$
\omega^{s}=A^{s} P^{s}\left(\mathrm{O}_{2}\right) \exp \left(\frac{-E_{a}^{s}}{R T_{p}}\right)
$$

where, $T_{p}$ is particle temperature measured in Kelvin. $A^{s}=21 * 10^{3} \frac{\text { mole }}{\mathrm{m}^{2} \cdot s \cdot a t m}$ is the pre-exponential factor, and $E_{a}^{s}=80 \mathrm{~kJ} /$ mole is the activation energy, as specified by the experiments [24]. Using Eq. 17, the reaction rate of each species at the surface is given as,

$$
\omega_{i}^{\prime}=\beta_{i} \operatorname{Da}^{s} Y_{2}^{s} \frac{\bar{W}}{\mathcal{W}_{\mathrm{O}_{2}}},
$$

where, $\bar{W}$ is the average molecular weight, $\left(\beta_{i}\right)_{i \in\{1,4\}}=\left([1-\psi] \mathcal{W}_{\mathrm{CO}},-\frac{1+\psi}{2} \mathcal{W}_{\mathrm{O}_{2}}, \psi \mathcal{W}_{\mathrm{CO}_{2}}, 0\right)$, and the surface Damköhler number $D a^{s}$ is,

$$
\mathrm{Da}^{s}=A^{s} \frac{C_{p} a}{\lambda} \exp \left(\frac{-E_{a}^{s *}}{T^{s *}}\right) P_{0} .
$$

Non-dimensionalisation has been performed as described in section 2.1. Finally, the solution satisfies the species conservation equation at the particle surface,

$$
\sum_{i=1}^{n=4} Y_{i}^{s}=1
$$

\subsection{Remaining boundary conditions}

Away from the particle, composition $\left(Y_{\mathrm{CO}}^{\infty}, Y_{\mathrm{O}_{2}}^{\infty}, Y_{\mathrm{CO}_{2}}^{\infty}\right.$ and $\left.Y_{\mathrm{H}_{2} \mathrm{O}}^{\infty}\right)$ and temperature $\left(T^{\infty}\right)$ are set. 


\section{Numerical method}

In its first-order form, the two-point boundary value problem, described in section 2 may be solved numerically using well-documented techniques, such as collocation or shooting methods. The latter was used in this work: it consists in solving the original problem as an initial value problem using readily available ODE integrators. The integration is performed outward from the particle surface but, since initial data such as mixture composition are missing, it is estimated and iteratively updated until the far field conditions are matched. The iterative solver employed in this work is the Levenberg-Marquardt algorithm, which proved able to handle all the cases presented in the results section (for more information regarding the numerical procedure refer to [8]).

\section{Adjoint-based methodology}

Traditionally, adjoint equations are derived from the continuous equations by applying a variational principle to the unconstrained optimisation problem and setting the first variations with respect to all involved dependent variables to zero. This results in governing equations for the direct (original) and for the adjoint variables, together with appropriate boundary conditions, initial conditions, and optimality expressions, which subsequently have to be discretized and implemented [12,2]. This approach is most commonly referred to as differentiate-then-discretize or continuous adjoint approach, and in particular, for complex governing equations and/or optimisation objectives, can become very cumbersome and error-prone. Alternatively, the spatially discretized equations (e.g. resulting from the application of the method of lines) can be used and processed by automaticdifferentiation $(\mathrm{AD})$ software to produce the associated adjoint code. This approach, on the other hand, is otherwise referred to as discretize-then-differentiate or discrete adjoint formalism. Due to the superior performance of the discrete adjoint methodology [8, when applied to system of equations similar to those described in section 2 it has been selected to extract the sensitivities in this study.

\subsection{Discrete adjoint formulation}

Following the approach of discretize-then-differentiate, the discrete adjoint equations are extracted directly from the discretized form of the primal problem, where linearisation is performed using complex step differentiation. It should be noted that since the discrete adjoint equations are extracted from the primal problem after the discretization is performed, the adjoint and the primal grids are identical. This is one of the main differences between the continuous and the discrete approaches [8], applied to this problem.

\section{Results}

In this section, sensitivities of multiple quantities of interests with respect to existing model/physical parameters are extracted. This study mainly focuses on the sensitivities with respect to the heterogeneous model parameters governing the reaction on the surface of the particle, as described in section 2. The variation of model sensitivities are then examined as particle size, particle temperature, and free stream composition are varied as specified in the experiments [24].

As particle size and shape are crucial parameters for reaction kinetics determination, two measurement techniques were employed to reduce uncertainty [29]: (i) Stereoscopic Camera system for Optical Thermography (SCOT), which is based on two-color pyrometry and (ii) light microscopy (Zeiss Axiophot, image scale $1.375 \mu \mathrm{m} /$ pixel), which allows a much easier sample handling and a higher accuracy due to the higher resolution and the stationary fixation of the particles on a flat 
surface. Particle surface temperature was also measured using two-color pyrometry at $\lambda_{1}=785$ and $\lambda_{2}=650$ micrometers, where similar measures were taken to reduce the uncertainty of measurements $(\approx 5 \%)$. Finally gas flow controllers provide accurate measurement of the reaction atmosphere.

Due to access to detailed and accurate measurement data, the adjoint-based analysis can be used to numerically provide sensitivities with respect to each measured model parameter, at each measurement location. Using the simulations to study the evolution of these sensitivities as free stream and/or particle conditions change, a trend can be extracted for the variation of sensitivities of the experimentally measured model parameters. For this propose, eight different experimental conditions are considered and analysed as shown in table 1. The symbols are used to identify the measured data in all the figures of this section. Note that the particle temperature, $T_{p}$ and free stream temperature (gas temperature in the experiment), $T^{\infty}$, are reported in non-dimensional form in the table.

\begin{tabular}{c|c|c|c|c|c|c|c|c} 
Case & $\mathrm{Y}^{\infty} \mathrm{O}_{2}$ & $\mathrm{Y}^{\infty} \mathrm{CO}_{2}$ & $\mathrm{Y}^{\infty} \mathrm{H}_{2} \mathrm{O}$ & $T_{p}$ & $T^{\infty}$ & $2 \times a[\mu m]$ & $\mathrm{Da}^{g}$ & Symbol \\
\hline 1 & 0.0796 & 0.8756 & 0.04477 & 0.1866 & 0.1623 & 121 & 1537 & $\bullet$ \\
2 & 0.0851 & 0.8191 & 0.09574 & 0.1820 & 0.1570 & 130 & 1800 & $\bullet$ \\
3 & 0.1641 & 0.7897 & 0.04615 & 0.2104 & 0.1717 & 125 & 1643 & $\square$ \\
4 & 0.1758 & 0.7252 & 0.09890 & 0.2051 & 0.1646 & 120 & 1520 & $\square$ \\
5 & 0.2539 & 0.6984 & 0.04761 & 0.2253 & 0.1762 & 123 & 1588 & $\circ$ \\
6 & 0.2727 & 0.6250 & 0.10227 & 0.2248 & 0.1654 & 125 & 1651 & $\circ$ \\
7 & 0.3497 & 0.6010 & 0.04918 & 0.2402 & 0.1724 & 107 & 1202 & $\triangle$ \\
8 & 0.3764 & 0.5176 & 0.10588 & 0.2375 & 0.1659 & 111 & 1299 & $\triangle$
\end{tabular}

Table 1: Experimental conditions, considered here for analysis.

Before analysing the evolution of model sensitivities, the effect of the choice of the quantity of interest (QoI) on the sensitivities, at a given measurement point (case 1 of table 1), is determined. For this purpose, sensitivities of representative model/physical parameters are considered. By comparing the sensitivities of the heterogeneous model parameters to the sensitivities in free stream compositions and particle temperature, one can estimate the impact of the accuracy of measurements on the prediction of the quantity of interest. Due to large variation in their values, absolute values of the extracted sensitivities are plotted in logarithmic scale. The selected quantities of interest are: (i) the total heat release (integrated in the spatial domain), (ii) burning rate, and (iii) $\psi$, given by equation 14. Figure 1 shows the distribution of the extracted sensitivities for the identified QoIs. Comparing the sensitivities of the parameters determining the value of the heterogeneous reaction: $A^{s}, E_{a}^{s}$, and $T_{a}$, suggests that the activation energy $E_{a}^{s}$ is generally the most sensitive parameter, as opposed to the pre-exponential factor, $A^{s}$, having the lowest sensitivities. $T_{a}$ dominates the extracted sensitivities for $\psi$, which is also expected, as it appears directly in the expression defining this quantity (equation 14. While, the activation energy shows high sensitivities, depending on the QoI, other physical parameters, such as the temperature at the particle surface, $T_{p}$, can contribute to comparable and sometimes larger sensitivities, suggesting that particle temperature needs to be measured extremely accurately, for the sensitivities with respect to model parameters to be meaningful.

As far as experimental observations are concerned, several studies have reported on the uncertainties in particle temperature measurements for burning char particles. Murphy \& Shaddix 18 use a ratio-pyrometer based on photo-multiplier tubes (PMT). The reported uncertainty in a single particle temperature measurement is reported to be better than $1 \%(\approx 20 \mathrm{~K})$ for typical char combustion conditions. Bejarano \& Levendis used another type of PMT-based three-color pyrometer 


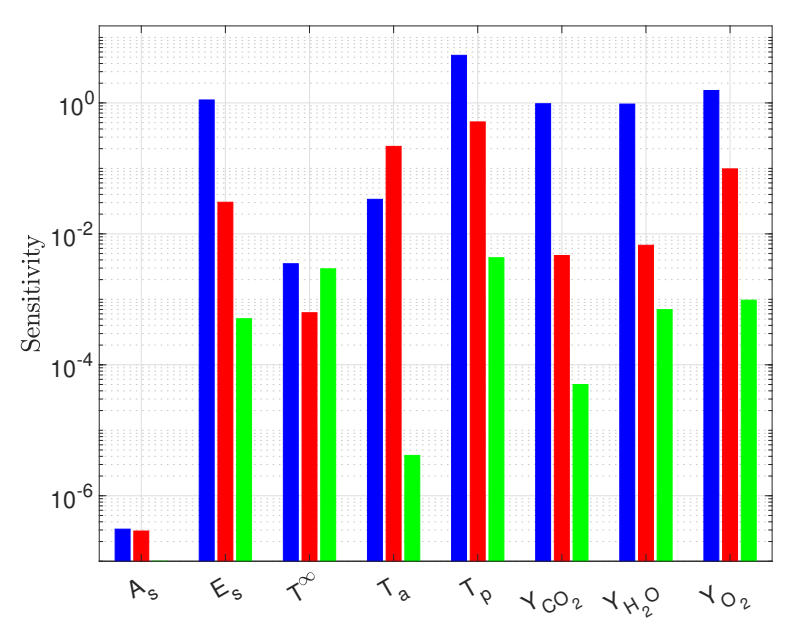

Fig. 1: The sensitivity of different quantities of interest with respect to existing model/physical parameters. (green), total heat release; (red), $\psi$; (blue) burning rate.

and investigated classy carbon spheres, which were supposed to be of relatively constant quality [1]. They observed a standard deviation of $70 \mathrm{~K}$ and some particle-to-particle variations, such that the systematic error in the temperature measurement is certainly smaller than this value. The technique adopted for experimental measurements of this study, is based on high-speed intensified CCD cameras (a very detailed analysis of the temperature uncertainty of single particle measurements, using this method, is given in [29]). Although this work reports an average uncertainty of up to $4.7 \%$ for all particles which are accepted for further evaluation, recording of several hundred particles at one residence time, or conversion level, reduces the error in the mean temperature. This leads to comparably low uncertainties in the average temperature, which is used for further calculations, e.g. the determination of reaction rate parameters (a study of the influence of the measurement uncertainty is given in [24]).

Figure 1 also shows that the extracted sensitivities are directly dependent on the choice of QoI. While one QoI might lead to high sensitivities with respect to a model parameter or physical compositions of the problem, another QoI might show no sensitivities at all. Therefore, if the QoI is not chosen correctly, it might have misleading consequences in the interpretation of the extracted sensitivities. In case no sensitivities are reported with respect to a certain QoI for a certain parameter, it should not be concluded that the overall system is independent on the parameter, just the prediction of the selected QoI.

Since the main objective of this study is determining the sensitivities in kinetic model parameters, the evolution of the extracted sensitivities with respect to the model parameters are examined, as the conditions specified in the free stream and at the particle surface change according to the values reported by the experimental measurements. The evolution is defined such that, the parameters of each experimental configuration is fixed as specified by table 1, except for the particle diameter, which is allowed to vary, as shown in figure 2. The symbols show the corresponding measurement location of each experiment. This figure, also compares the evolution of sensitivities extracted for all heterogeneous model parameters: $A_{s}, E_{a}$, and $T_{a}$, although the activation energy $E_{a}$ remains the most sensitive throughout. Considering the experimental conditions (symbols in the figures), independent of the model coefficient, case 8 results in the highest sensitivities, and case 1 leads to the lowest, as far as the prediction of the burning rate is concerned. The burning rate, using the heterogeneous model presented in section 2 , is directly related to the global reaction 


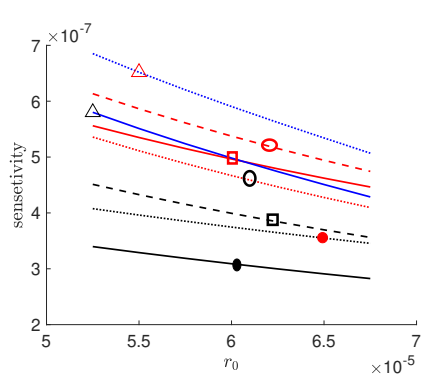

(a) $A^{s}$

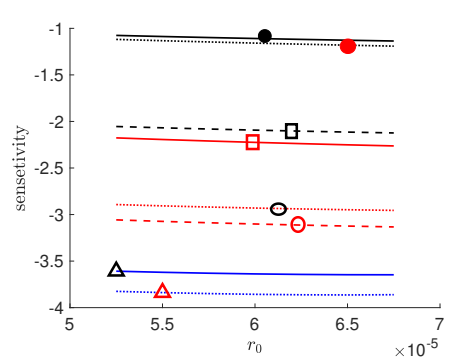

(b) $E_{a}^{s}$

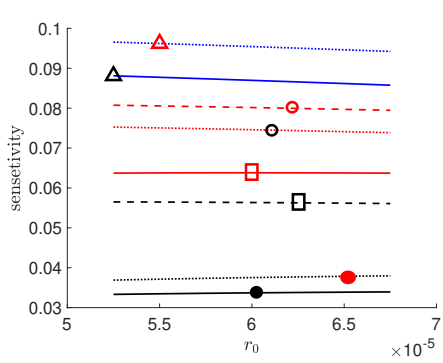

(c) $T_{a}$

Fig. 2: Evolution of model sensitivities with respect to particle size (radius), for burning rate as QoI: $-\& \bullet$, case $1 ; \cdots \& \bullet$, case $2 ;---\& \square$, case $3 ;-\& \square$, case $4 ; \cdots \& \circ$, case $5 ;---\&$ $\circ$, case $6 ;-\& \triangle$, case $7 ; \cdots \& \triangle$, case 8 . Lines: simulations, where the free stream and particle conditions are fixed similar as specified by the experiment, and particle size is allowed to vary; Symbols: experimental conditions.

rate, described by equation 17 , which in turn depends on the oxygen partial pressure at the surface, $P^{s}\left(\mathrm{O}_{2}\right)$. Increasing the oxygen concentration in the free stream, results in higher concentrations at the surface as well, strengthening the effect of the surface reaction, which leads to higher sensitivities in prediction of the burning rate. On the other hand, experiments have shown [29, that higher oxygen concentrations lead to higher particle temperatures, which in turn reduce uncertainties in measurements. More accurate measurements of surface temperature, may compensate the higher sensitivities reported here. Confirmation of this fact requires further research, beyond the scope of this work. When studying the evolution of sensitivities with respect to particle size (lines in figure 2), experimental condition imposed through case 8, still produces the highest sensitivities of the burning rate. This figure also suggests that particle size has a weak impact on the sensitivities of the pre-exponential factor (figure 2(a)), with smaller particles having slightly higher sensitivities. However, the effect of particle size is negligible as far as activation energy and $T_{a}$ are concerned, figures 2(b) and 2(c), respectively. Since these two parameters show higher sensitivities in prediction of the burning rate than the pre-exponential factor, $A^{s}$, it can be concluded that, the particle size has a minor effect on the extracted sensitivities of the most dominant model parameters.

Figure 1 shows that, as far as the burning rate is concerned, the dominant parameter regarding the prediction of its value, could indeed be the particle temperature or the free stream composition $\left(\mathrm{Y}_{\mathrm{CO}_{2}}\right.$ is selected here as a representative, other quantities such as oxygen mass fraction follow the same trend). As a result, figure 3 shows the evolution of sensitivities extracted with respect to these two parameters. Comparison of figure 3(a) and 2(b) shows that the sensitivities with respect to the particle temperature follow the same trend as that of the activation energy $E_{a}^{s}$. On the other hand, the sensitivities of $\mathrm{Y}_{\mathrm{CO}_{2}}$ follow the opposite trend, with highest sensitivities reported for case 1. A closer inspection of this case shows higher concentrations of $\mathrm{Y}_{\mathrm{CO}_{2}}$ in the experimental condition as well, which could explain the impact on the extracted sensitivities. The sensitivities with respect to particle temperature is higher for all the considered cases compared to the other existing model/physical parameters, with the sensitivities with respect to the activation energy of the heterogeneous reaction following closely. This results confirm the conclusion, that for all experimental conditions considered here, particle temperature should be measured accurately to allow sensitivity analysis of the model parameters.

Here, the impact of the choice of QoI on the evolution of sensitivities is examined, and instead of the burning rate, the total heat release is selected as the desired output. The evolution of sensitivities, extracted for this QoI, is shown in figure 4 Figure 1 showed that, while the activation 


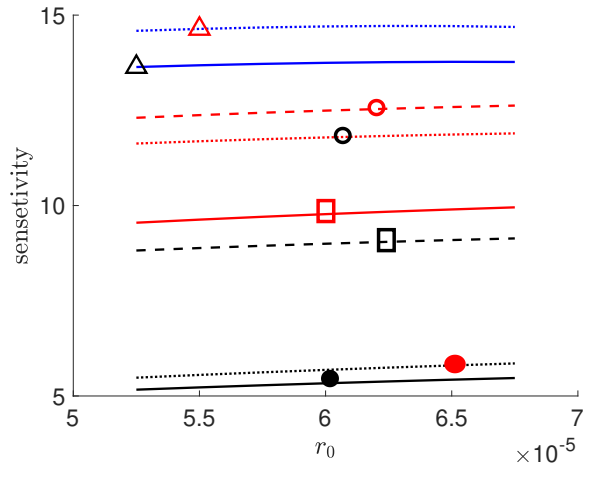

(a) $T_{p}$

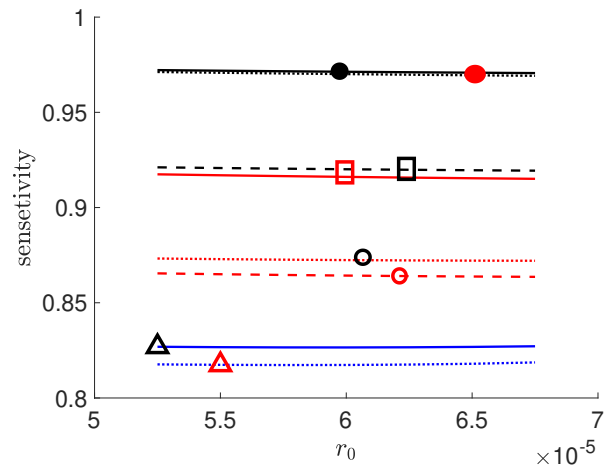

(b) $\mathrm{Y}_{\mathrm{CO}_{2}}$

Fig. 3: Evolution of sensitivities for $T_{p}$ and $\mathrm{Y}_{\mathrm{CO}_{2}}$ with respect to particle size (radius), for burning rate as QoI: $-\& \bullet$, case $1 ; \cdots \& \bullet$, case $2 ;---\& \square$, case $3 ;-\& \square$, case $4 ; \cdots \& \circ$, case 5 ; $---\& \circ$, case $6 ;-\& \triangle$, case $7 ; \cdots \& \triangle$, case 8 . Lines: simulations, where the free stream and particle conditions are fixed similar as specified by the experiment, and particle size is allowed to vary; Symbols: experimental conditions.

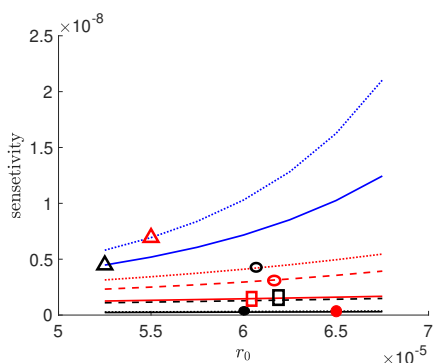

(a) $A^{s}$

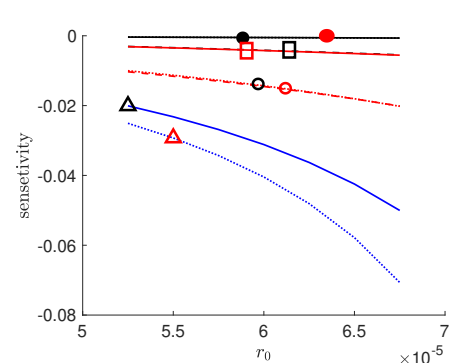

(b) $E_{a}^{s}$

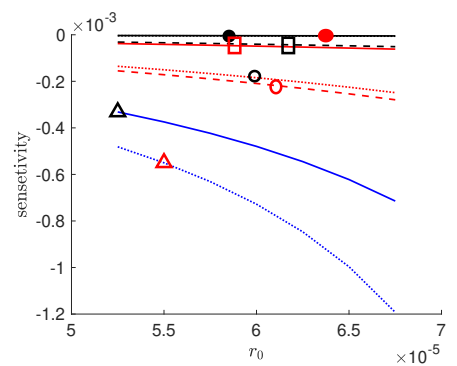

(c) $T_{a}$

Fig. 4: Evolution of model sensitivities with respect to particle size (radius), for total heat release as QoI: $-\& \bullet$, case $1 ; \cdots \& \bullet$, case $2 ;---\& \square$, case $3 ;-\& \square$, case $4 ; \cdots \& \circ$, case $5 ;---\&$ $\circ$, case $6 ;-\& \triangle$, case $7 ; \cdots \& \triangle$, case 8 . Lines: simulations, where the free stream and particle conditions are fixed similar as specified by the experiment, and particle size is allowed to vary; Symbols: experimental conditions.

energy $E_{a}^{s}$ remains the most sensitive model parameter for both quantities of interest, burning rate results in higher sensitivities compared to total heat release. This proves to be the case for all the experimental conditions that are considered here, as comparison of figures 4(b) and 2(b) suggests. Similar to the case of the burning rate, the highest sensitivities are calculated for case 8 and the lowest for case 1 . The higher oxygen concentration together with higher particle temperature in cases 7 and 8, result in higher temperatures throughout the domain, increasing the sensitivities of prediction as far as the heat release is concerned. In contrast to figure 2, however, the particle size has a larger effect on the extracted sensitivities, with larger particle sizes leading to higher sensitivities. For cases with highest sensitivities, cases 7 and 8 , the effect of particle size is also more substantial. The pronounced dependence on particle size is due to the particular definition of $\mathrm{Da}^{g}$ (equation 10), used in this model, which results in quadratic dependence on the particle 


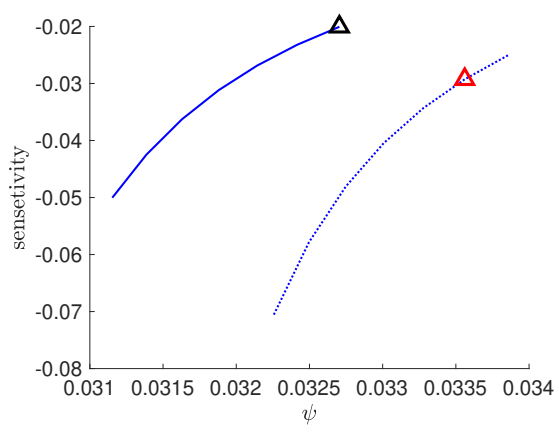

Fig. 5: Evolution of sensitivities in $E_{a}^{s}$, with respect to $\psi$, for heat release as QoI:- \& $\triangle$, case 7 ; $\cdots \& \triangle$, case 8

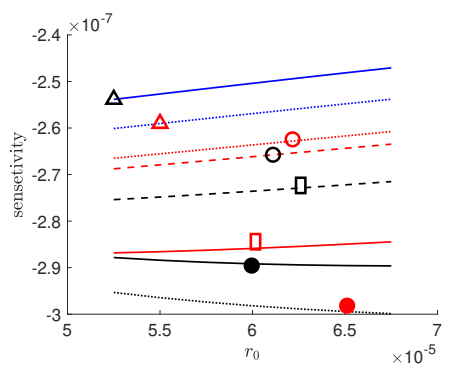

(a) $A^{s}$

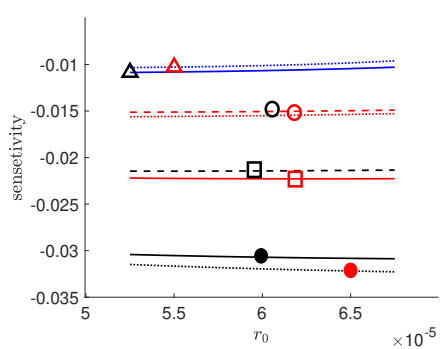

(b) $E_{a}^{s}$

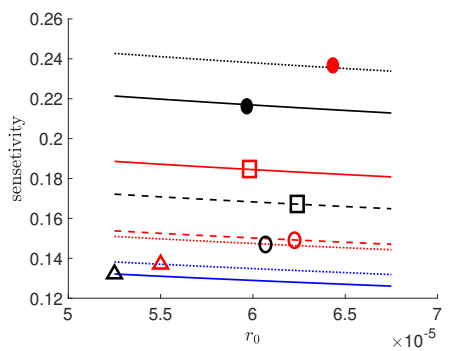

(c) $T_{a}$

Fig. 6: Evolution of model sensitivities with respect to particle size (radius), for $\psi$ as QoI: — \& • case $1 ; \cdots \& \bullet$, case $2 ;---\& \square$, case $3 ;-\& \square$, case $4 ; \cdots \& \circ$, case $5 ;---\& \circ$, case $6 ;-\& \triangle$, case $7 ; \cdots \& \triangle$, case 8 . Lines: simulations, where the free stream and particle conditions are fixed similar as specified by the experiment, and particle size is allowed to vary; Symbols: experimental conditions.

radius. Note that the total heat release is calculated by integrating the reaction rate in the whole spatial domain, containing the gas phase. As a result, although the sensitivities are extracted with respect to the surface parameters, the behaviour is dependant on the dynamics, prescribed by both heterogeneous and homogeneous reactions.

As described in detail by Schiemann et al. 24], $\psi$ is an important parameter in the calculation of rate parameters $A_{s}$ and $E_{a}^{s}$. As a result, figure 5 shows the evolution of the sensitivities with respect to $\psi$, replacing particle size. This figure includes only the evolution of the sensitivity with respect to the most dominant parameter $E_{a}^{s}$, and for the experimental setup of case 8 and 7 , which were the most sensitive (figure 4(b)). Comparing figures 4(b) and 5 shows that, plotting versus $\psi$ rather than particle radius changes the trend in the evolution of sensitivities. Lower values of $\psi$ result in higher sensitivities, suggesting an inverse relationship between the value of $\psi$ and the particle size. This inverse proportionality can be explained by considering the definition of the $\mathrm{Da}^{s}$ in equation 19. This equation shows the direct relation between the particle size and $D a^{s}$. Increasing particle size increases $D a^{s}$, resulting in faster reactions, and higher carbon dioxide production, hence reducing the oxygen mass fraction on the surface, and in turn, reducing the value of $\psi$, defined by equation 14 . Therefore, all the plots reported for particle size can be interpreted similarly for $\psi$, considering this inverse proportionality. 
Figure 6 shows the evolution of sensitivities extracted for $\psi$ as the quantity of interest. As demonstrated by equation 14. this variable is one of the necessary parameters in determining the reaction rate of the heterogeneous reaction, and is also the solution of the equations governing the steady char combustion, explained in section 2. In contrast to the other quantities of interest, $T_{a}$ is the most sensitive parameter as far as the prediction of $\psi$ is concerned. Another difference to the other QoIs is that, higher sensitivities are reported for case 1 as opposed to case 8. This is expected, since $T_{a}$ appears directly in the definition of $\psi$ (equation 14). Considering the form of the equation, it is obvious that the sensitivity of $\psi$ is adversely related to the partial pressure of oxygen, favoring case 1, where lower free stream oxygen concentrations are imposed by the experimental conditions. Similar to the burning rate, however, there seems to be a week dependence on the particle size, and this could be attributed to the dominance of the heterogeneous reaction in determining the value of this parameter.

As a final remark, it should be noted that, while extracted sensitivities are important in assessing and interpreting model/flow dynamics, they are, in effect, a linear local interpretation of the response of the underlying system to slight variations in model or flow parameters. For a nonlinear alternative, uncertainty quantification should be used. In experimental studies, the results are commonly reported with error bars indicating the level of uncertainties in the measurements. In order to provide similar information from simulations, the extracted sensitivities can be used to approximate such uncertainty information. Such analysis will be the subject of future work for this study.

It should be also noted that, the extracted sensitivities are directly dependent on the choice of the kinetic model used to perform the simulations. Despite well-known weaknesses of the onestep heterogeneous model analysed here, it is commonly used in literature to give a quantitative description of different experimental or fuel-dependent parameters on the resulting reactivity, motivating its use in the experimental and numerical investigations here. However, a variety of other char combustion models exist, many of them consider more physical and chemical effects than the one-step kinetics model, which has been applied here (for example, the CBK model family [10, 20], and Senneca's model [26], to name a few). Although different in their overall structures, these models all consider multiple chemical reactions for char conversion, coupled serially and/or in parallel. Nevertheless, the model calibration to a fuel relies on a single or a few parameters of specific reactions, and the remaining parameters are defined by fixed ratios in these models. Due to the different structure of these models, the sensitivity of the model performance might not be equal to the presented case, but similar tendencies are expected. These models are far more complicated in their mathematical structure, therefore a detailed analysis of the sensitivities of the involved parameters is left to later work.

\section{Summary and conclusions}

This study focuses on a heterogeneous model, commonly implemented in numerical simulations, in order to predict the reaction process on the surface of a solid particle (in this case char particle), during the combustion process. The particular model of interest to this project is extracted through detailed measurement of Schiemann et al. 24], where model parameters are reported for various, particle sizes, free stream compositions, and particle temperatures. Using adjoint-based methodology, sensitivities of the model parameters are extracted for various quantities of interests, including the burning rate and the total heat release. Finally, the variation of the sensitivities are reported as the particle size, and free stream compositions are varied, as specified by the experimental measurements. The analysis shows the activation energy to be the most sensitive parameter, as far as, the burning rate and total heat release are concerned. It should be noted, however, that particle temperature can also result in comparable sensitivities, when approximating the burning rate. The resulting parameter sensitivities vary slightly with particle diameter for burning rate as 
QoI. On the other hand, particle size shows a much higher impact in the extracted sensitivities when considering the total heat release. This is attributed to the fact that the total heat release is an integrated quantity, evaluated within the entire domain, whereas the burning rate is a local quantity determined mainly by the heterogeneous reaction rate on the surface of the particle. The analysis shows that the free stream composition has a large impact in the extracted sensitivities. Free stream conditions with higher oxygen concentrations, generally result in higher sensitivities in the model coefficients. Higher oxygen concentration leads to stronger reactions on the surface, increasing the burning rate, leading to higher sensitivities in the model parameters as well.

Acknowledgements The authors kindly acknowledge financial support through Deutsche Forschungsgemeinschaft (DFG)- Projektnummer 215035359 - TRR 129.

\section{References}

1. Bejarano, P., Levendis, Y.: Combustion of Coal Chars in Oxygen-Enriched Atmospheres. Combust. Sci. Technol. 179, 1569-1587 (2007)

2. Bewley, T., Moin, P., Temam, R.: Dns-based predictive control of turbulence: an optimal benchmark for feedback algorithms. J Fluid Mech. 447, 179-225 (2001)

3. Braman, K., Oliver, T., Raman, V.: Adjoint-based sensitivity analysis of flames. Comb. Theo. Modeling 19(1), 29-56 (2015)

4. Essenhigh, R.: Predicted burning times of solid particles in an idealized dust flame. J Inst. Fuel 34, 239-244 (1961)

5. Foures, D., Caulfield, C., Schmid, P.: Optimal mixing in two-dimensional plane poiseuille flow at finite péclet number. J Fluid Mech. 748, 241-277 (2014)

6. Freund, J.: Adjoint-based optimization for understanding and suppressing jet noise. Procedia Engineering 6, 54-63 (2010)

7. Giles, M., Pierce, N.: An introduction to the adjoint approach to design. Flow Turb. Combust. 65(3), 393-415 (2000)

8. Hassan, A., Sayadi, T., Chenadec, V.L., Pitsch, H., Attili, A.: Adjoint-based sensitivity analysis of steady char burnout. Comb. Theo. Modeling (under review) (2020)

9. Hecht, E., Shaddix, C., Lighty, J.: Analysis of the errors associated with typical pulverized coal char combustion modeling assumptions for oxy-fuel combustion. Combust. Flame 160(8), 1499-1509 (2013)

10. Holland, T., Fletcher, T.: Comprehensive model of single particle pulverized coal combustion extended to oxy-coal conditions. Energy Fuels 31, 2722-2739 (2017)

11. Howard, J., Williams, G., Fine, D.: Kinetics of carbon monoxide oxidation in postflame gases. Fourteenth Symposium (International) on Combustion pp. 975-986 (1973)

12. Jameson, A.: Aerodynamic shape optimization using the adjoint method. Lectures at the Von Karman Institute (2003)

13. Juniper, M.: Triggering in the horizontal rijke tube: non-normality, transient growth and bypass transition. J Fluid Mech. 667, 272-308 (2010)

14. Lemke, M., Cai, L., Reiss, J., Pitsch, H., Sesterhehn, J.: Adjoint-based sensitivity analysis of quantities of interest of complex combustion models. Comb. Theo. Modeling 23(1), 180-196 (2019)

15. Lemke, M., Reiss, J., Sesterhenn, J.: Adjoint-based analysis of thermoacoustic coupling. ICNAAM pp. 2163$2166(2013)$

16. Matalon, M.: Complete burning and extinction of a carbon particle in an oxidizing atmosphere. Fourteenth Symposium (International) on Combustion 24, 115-127 (1980)

17. McConnell, J., Sutherland, J.: The effect of model fidelity on prediction of char burnout for single-particle coal combustion. Proc. Combust. Inst. 36(2), 2165-2172 (2016)

18. Murphy, J., Shaddix, C.: Combustion kinetics of coal chars in oxygen-enriched environments. Combust. Flame 144, 710-729 (2006)

19. Nemili, A., Ozkaya, E., Gauger, N.R., Kramer, F., Thiele, F.: Discrete adjoint based optimal active control of separation on a realistic high-lift configuration. New Results in Numerical and Experimental Fluid Mechanics X: Contributions to the 19th STAB/DGLR Symposium Munich, Germany, 2014. Springer International Publishing pp. 237-246 (2016)

20. Niksa, S., Liu, G., Hurt, R.: Coal conversion submodels for design applications at elevated pressures. Part I. Devolatilization and char oxidation. Prog. Energy Combust. Sci. 29, 425-477 (2003)

21. Nusselt, W.: Der verbrennungsvorgang in der kohlenstaubfeuerung. Z Ver Deut Ing 68, 124-128 (1924)

22. Rabin, S., Caulfield, C., Kerswell, R.: Designing a more nonlinearly stable laminar flow via boundary manipulation. J Fluid Mech. 738(R1), 1-12 (2014) 
23. Sashittal, P., Sayadi, T., Schmid, P., Jang, I., Magri, L.: Adjoint based sensitivity analysis for a reacting jet in crossflow. Proceeding of the Center of Turbulence Research summer program pp. 375-284 (2016)

24. Schiemann, M., Geier, M., Shaddix, C., Vorobiev, N., Scherer, V.: Determination of char combustion kinetics parameters: Comparison of point detector and imaging-based particle-sizing pyrometry. Rev. Sci. Instruments $85(2014)$

25. Schmidt, S., Ilic, C., Schulz, V., Gauger, N.R.: Three-dimensional large-scale aerodynamic shape optimization based on shape calculus. AIAA J 51(11), 2615-2627 (2013)

26. Senneca, O., Salatino, P.: A semi-detailed kinetic model of char combustion with consideration of thermal annealing. Proc. Combust. Inst. 33, 1763-1770 (2011)

27. Spalding, D.: Combustion of fuel particles. Fuel 30, 239-244 (2016)

28. Tognotti, L., Longwell, J., Sarofim, A.: The products of the high temperature oxidation of a single char particle in an electrodynamic balance. Proc. Combust. Inst 24, 1207-1213 (1991)

29. Vorobiev, N., Valentiner, S., Schiemann, M., Scherer, V.: Comprehensive data set of single particle combustion under oxy-fuel conditions, part i: Measurement technique. Combus. Sci. Tech. pp. 1-22 (2020) 